\title{
411 - Palliative Care in Dutch Green Care Farms and Innovative Small-Scale Living Facilities
}

\author{
Authors: Brittany DeGraves, Judith Meijers, Carole Estabrooks, Hilde Verbeek
}

Background: Dutch Green Care Farms and innovative small-scale facilities for residents with dementia focus on promoting resident abilities and engaging residents in activities to maintain resident quality of life up until death. Palliative care approaches are needed in these innovative facilities to maintain quality of life and quality of care. To our knowledge, there are currently no literature reviews discussing palliative care in these facilities or exploring palliative care in Green Care Farms in the Netherlands.

Research Objectives: To identify current palliative care approaches at innovative small-scale facilities and Dutch Green Care Farms for residents with dementia.

Methods: This two-part study includes a scoping review focused on identifying research regarding palliative care approaches in innovative small-scale facilities. Thematic analysis based on van der Steen et al.'s (2014) domains for palliative care in dementia were used to analyze the final papers included in the review. The second part of the study included qualitative semi-structured interviews of six managers and two front-line caregivers at five care farms in the Netherlands in 2019 focused on palliative care approaches in their facilities.

Preliminary Results of Ongoing Study: Eight papers were included in our final scoping review. Most facilities in these papers focused on maximizing comfort and the residents remaining abilities at end-oflife while providing person and family-centred care. Similar findings were seen in the preliminary results of the interviews, which indicate that all farms use palliative care approaches while maximizing physical and psychological functioning and engagement in activities. Care farms also reported promoting family involvement, preventing hospitalizations, and ensuring family and resident involvement in decisionmaking at the end-of-life.

Conclusion: By providing palliative care approaches, innovative facilities provide a home for life and improved quality of life for residents up until death. Innovative small-scale facilities including green care farms prioritize resident autonomy and choice, minimizing discomfort and family-centred care at the end-of-life. Additional research surrounding barriers to palliative care, quality of end-of-life care, and comparisons of innovative facilities to traditional long-term care homes are needed to have a more comprehensive picture of palliative care in these innovative facilities.

\section{2 - Empowerment for people living with dementia: an integrative literature review \\ Charlotte T.M. Van Corven, Annemiek Bielderman, Mandy Wijnen, Ruslan Leontjevas, Peter LBJ Lucassen, Maud JL Graff, Debby L. Gerritsen, PhD}

Introduction: Although the concept of empowerment seems useful for good care and support for people living with dementia, there is a lack of understanding as to how to define this concept. Therefore, insight is needed in what empowerment means for people living with dementia.

Methods: We performed an integrative literature review (PubMed, CINAHL, PsychINFO), including articles that addressed empowerment for people living with dementia in their title or abstract. Using qualitative data analysis software ATLAS.ti, we applied open codes to describe all relevant aspects of included articles. Common themes and categories were identified using inductive reasoning and constant comparison.

Results: Sixty-nine articles were included. We identified four themes: (1) description of the state of being empowered, (2) the process of empowerment, (3) contribution of the environment to the empowerment process, and (4) effects on other variables. We combined these results with the conceptual framework of our previous qualitative study on the definition of empowerment for people 